\title{
Ventilation-analogue mechanostimulation of lung epithelial cells in vitro
}

\author{
Gamerdinger K., Schumann S., Smudde E. and Guttmann J. \\ Division for Experimental Anesthesiology, University Medical Center Freiburg, Germany \\ katharina.gamerdinger@uniklinik-freiburg.de
}

\begin{abstract}
During spontaneous breathing the mechanical strain on the pulmonary tissue is akin to a sinusoidal profile. In contrast, during mechanical ventilation the stimulation profile of the lung tissue differs considerably from a sinusoidal pattern as it can be shown analyzing the frequency contents of the ventilatory pattern by means of a Fourier-Transformation. In vitro used methods for mechanostimulation of soft biologic tissue for the analysis of cellular reactions to dynamic stress or strain use exclusively sinusoidal stimulation patterns. In this work for the first time we compare reactions of lung epithelial cells in vitro stimulated either with sinusoidal or ventilation-analogue mechanostimulation profile. Our previously published mechanostimulator-system allows not only for stimulating the cells but thereby also for analyzing the mechanical properties of cell layers.
\end{abstract}

\section{Introduction}

Mechanical ventilation causes ventilation-induced lung injury (VILI). This is due to the fact that during mechanical ventilation cyclic alveolar recruitment/derecruitment occurs, which is associated with considerable mechanical shear stress in the alveolar parenchyma.

To analyze the effects of this stress to pulmonary cells several types of experimental mechanostimulation devices for in vitro applications of mechanical strain were established over the past years. However, most of these mechanical strain applicators mediate their mechanical load via a sinusoidal dynamic stimulation profile, thereby mimicking more or less a spontaneous breathing situation. The investigation of the mechanical ventilation associated dynamic effects on the isolated pulmonary cells requires an experimental mechanostimulator which is able to simulate the dynamics occurring during mechanical ventilation. The concern of this work was to establish an in vitro model that allows comparing the cellular reactions to sinusoidal stress application with cellular reactions to stress application mimicking the dynamics of mechanical ventilation.

Therefore we combined our previously established mechanostimulator with a linear motor drive which allows to mimick complex dynamic strain profiles. The drive system can apply an identical mechanostimulation pattern to four separate mechanostimulators at the same time. The unique features of our experimental mechanostimulator device are (i) the variability of the stimulation pattern including ventilation-analogue stimulation and (ii) the implemented pressure measuring sensor enabling the analysis of the mechanical properties of the cell layers.

\section{Methods}

In this work alveolar epithelial cells A549 were grown on RGD-coated, highly flexible polydimethyl siloxane (PDMS) membranes and were mechanically stimulated in the mechanostimulator. The confluent cell layers were subjected to sinusoidal or ventilation-analogue mechanical strain. During mechanostimulation the resulting pressure that the cells opposed to the applied strain was measured. Cellular integrity was determined using FACS analysis for apoptosis and/or necrosis staining

\subsection{Mechanostimulator}

We used a previously described mechanostimulator, for the mechanostimulation and analysis of mechanical properties of cell monolayers [1], [2]. The mechanostimulator consists of two chambers which are separated by a highly flexible impermeable silicone membrane on which the cells are grown. The bottom (pressure) chamber is connected on one side to a piston pump whose position is controlled by a linear motor. On the other side the pressure chamber is attached to a pressure measuring device. The top chamber serves as a supply chamber to provide nutrition fluid to the cells under mechanical stimulation.

\subsection{Stimulation profiles}

To analyze the frequency components of the cyclic mechanical stress which are transferred to the cell layer the mechanostimulator was driven with sinusoidal or ventilation-analogue profiles simulating the ventilatory pattern which is associated with the volume controlled ventilation (VCV). An amplitude of $1 \mathrm{~mL}$ volume, corresponding to an increase of surface area of $20 \%$, and a frequency of $15 / \mathrm{min}$ were chosen. 


\subsubsection{Ventilation-analogue profile}

The VCV-analogue mechanostimulation was realized to mimick constant inspiratory flow profile and exponentially decaying expiratory flow profile of a healthy lung with an expiratory time-constant of $400 \mathrm{~ms}$.

\subsection{Frequency Analysis}

To analyze the frequency components of the strain applied to the sample, the frequency spectrum of the pressure profile which resulted from the mechanostimulation was calculated using the FastFourier Transformation. From the resulting complex frequency spectrum the absolute values were calculated and normalized to the frequency component at the stimulation frequency (figure 1).
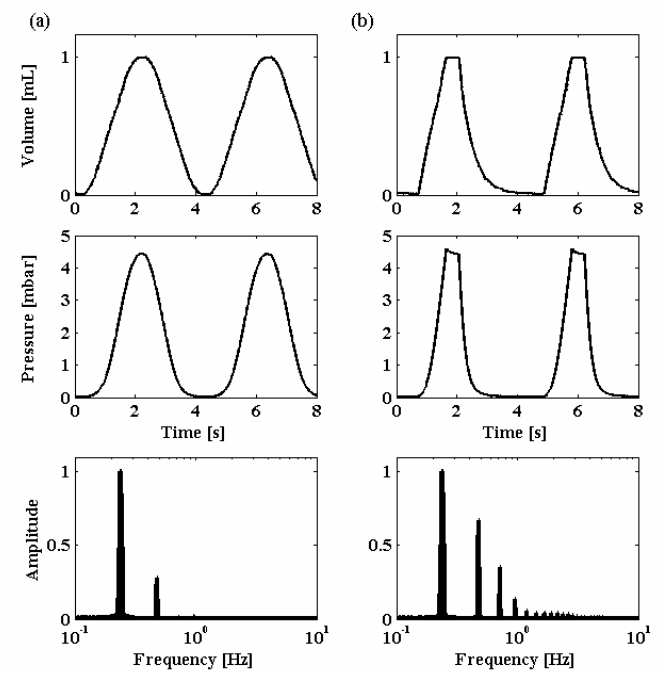

Figure 1 Frequency analysis of the stimulation patterns (average of 4 membranes)

(a) sinusoidal stimulation pattern (b) ventilation-analogue stimulation pattern. Top: insufflated volume over time. Middle: pressure data concerning the applied volume. Bottom: frequency components according to Fast-Fourier Transformation.

\subsection{Membrane production}

Membranes are produced in a spin-coating process as described in [2]. As membrane material we used PDMS. Base resin and curing agent were mixed in a weight ratio of 20:1. Rotation speed for spin-coating was $2.000 \mathrm{rpm}$ and rotation duration time was $1 \mathrm{~min}$, if nothing else is indicated.

\subsection{Membrane modification for cell seeding}

To reduce hydrophobicity of PDMS membranes and to allow cell adherence, membranes were modified as described by $\mathrm{Li}$, et al. [3]. The cured membrane on the carrier-ring was modified with $0.5 \mathrm{mg} / \mathrm{ml}$ Sulfo-SANPAH for 30min under UV-irradiation. Membranes were washed three times with water and three times with PBS. Afterwards membranes were incubated overnight with $1 \mathrm{mM}$ RGD-peptide solution. The other day, membranes were washed 3 times with PBS and kept with PBS on top, until the cells were prepared for seeding [3].

\subsection{Cell seeding}

For cell seeding on RGD-modified PDMS membranes, membranes were placed in culture rings in a six well plate and underlaid with the corresponding medium. We used alveolar epithelial cells A549. Afterwards $2.6 \cdot 10^{5} \mathrm{cells} / \mathrm{cm}^{2}$ in $1.3 \mathrm{ml}$ culture medium were spread to the center of the membranes. The plate was carefully transferred to the incubator to allow cell adherence and growth for at least $40 \mathrm{~h}$.

\subsection{Deflection protocol}

The membranes were warily removed from the culture rings and placed in membrane holders. To check cellular confluence three microscopic images per membrane were taken at defined positions. Subsequently membranes were placed in the mechanostimulator and deflection was started. Membranes were deflected for $10 \mathrm{~min}$ at room temperature, meanwhile pressure data were recorded. After deflection another 3 microscopic images were taken from equal positions on the membrane surface. Immediately after the procedure cells were trypsinized, sampled and stained for apoptosis and necrosis and analysed by FACS. Unstimulated cells on membranes served as negative controls.

\section{Results}

Stretching pulmonary A549 cells bidirectionally by sinusoidal deflection profile led to a change in mechanical properties equivalent to the surface increase. As shown in figure 2 the volume distensibility (compliance) of A549 cell layers markedly increased with increasing distensions. Remarkably, ventilation-analogue stimulation changed mechanically properties alike to the sinusoidal deflection (data not shown).

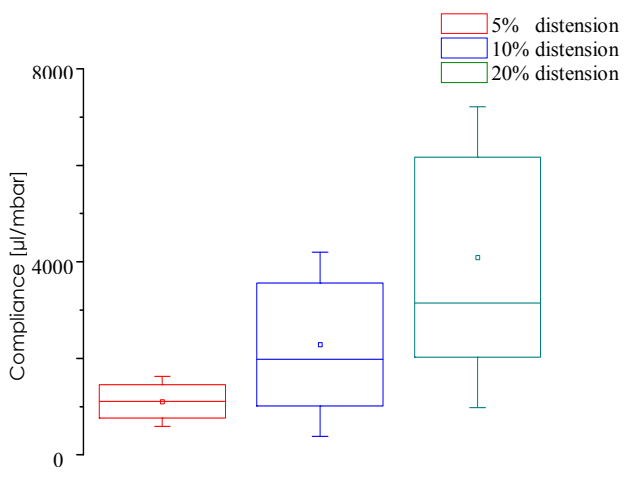

Figure 2 Compliance (volume distensibility) of A549 cell layers during sinusoidal mechanostimulation with a distension of either $5 \%, 10 \%$ or $20 \%$ surface increase.

After 10 minutes of sinusoidal or ventilation-analogue mechanostimulation microscopic images of the cellular layer on the membrane surface were taken. In figure 3 two 
typical examples are shown before and after stimulation with either a sinusoidal or a ventilation-analogue profile. Accordingly to the missing variances in mechanical behaviour, obvious differences in the cell layers after the different stimulations could not be found. Looking at the pictures, one would suggest that the mechanical integrity of the cell layer is a bit more reduced by the high frequency stimulation applying the ventilation-analogue profile compared to the sinusoidal pattern.

However, the analysis of apoptosis and necrosis rates after the mechanostimulation showed an increase in cell death after ventilation-analogue stimulation compared to sinusoidal stimulation as shown in figure 4. This increase in cell death is not only due to an increased necrosis rate, but also due to a significant increase in apoptosis rate, compared to the unstimulated or sinusoidally stimulated cells.

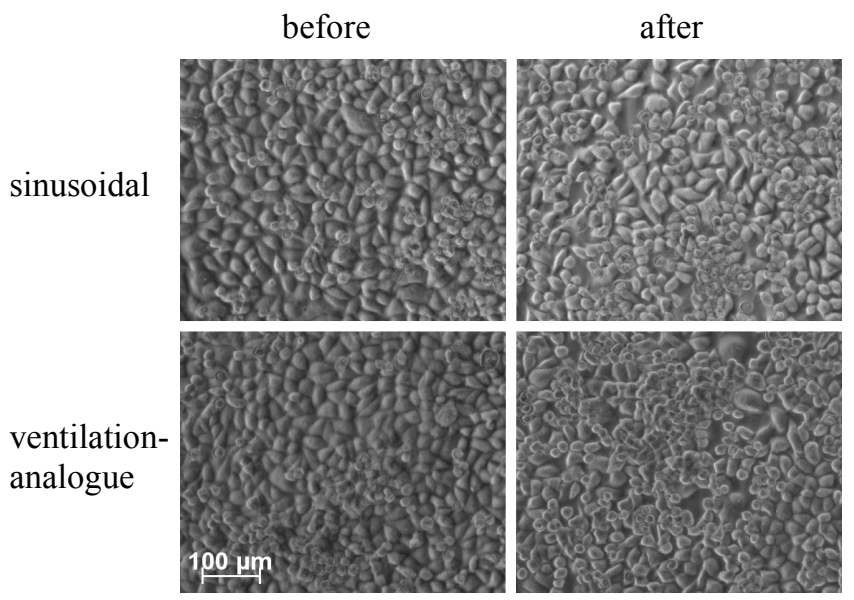

Figure 3 Alveolar epithelial cells (A549) before (left) and after (right) mechanostimulation. Top: Sinusoidal stimulation, bottom: ventilation-analogue stimulation.

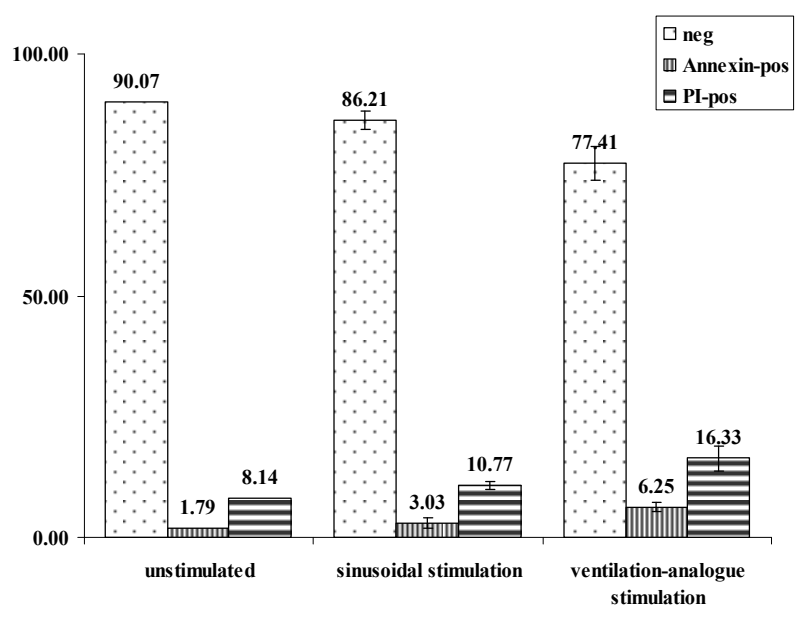

Figure 4 Increase of cell death after ventilation-analogue mechanostimulation shown by FACS analysis. The left bars show the percentages of live/dead unstimulated cells, the middle bars show the percentages of live/dead sinusoidally stimulated cells and the right bars show the percentages of live/dead cells after ventilation-analogue stimulation. Death rates are separately shown for apoptosis-induced (Annex-pos.) death or necrosis (PI-pos, Propidiumiodide).

\section{Conclusion}

Cell damages related to ventilation-analogue mechanostimulation as found in this study could play a role in development of ventilator induced lung injury.

\section{References}

[1] Schumann S, et al.: J Biomed Mater Res B Appl Biomater. 86: 483-92, 2008

[2] Armbruster C, et al.: J Biomed Mater Res B Appl Biomater. 91: 700-5, 2009

[3] Li, B., et al. J Biomed Mater Res A, 79: 989-98, 2006

\section{Acknowledgment}

This work was supported by the Deutsche Forschungsgemeinschaft Grant \# 561/GU 9-1

- Author: Katharina Gamerdinger

- Institute: Division for Experimental Anesthesiology

- $\quad$ Street: Hugstetter Strasse 55

- City: 79106 Freiburg

- Country: Germany

- Email:

- katharina.gamerdinger@uniklinik-freiburg.de 